\title{
$\operatorname{con} F-841117-11$
}

UCRL- 90935

PREPRINT

\section{LOW-ENERGY, HIGH-INTENSITY POSITRON BEAM EXPERIMENTS WITH A LINAC,}

\author{
R. H. Howe11, M. J. Fluss, I. J. Rosenberq \\ and $P$. Meyer \\ Lawrence Livermore National Labnratory \\ University of California \\ Livermore, CA 94550 \\ This paper was prepared for submittal to \\ 8 th Conference on the A.pnlication of \\ Accelerators in Research ind Industry \\ Denton, $T X$ \\ November 12-14, 1984
}

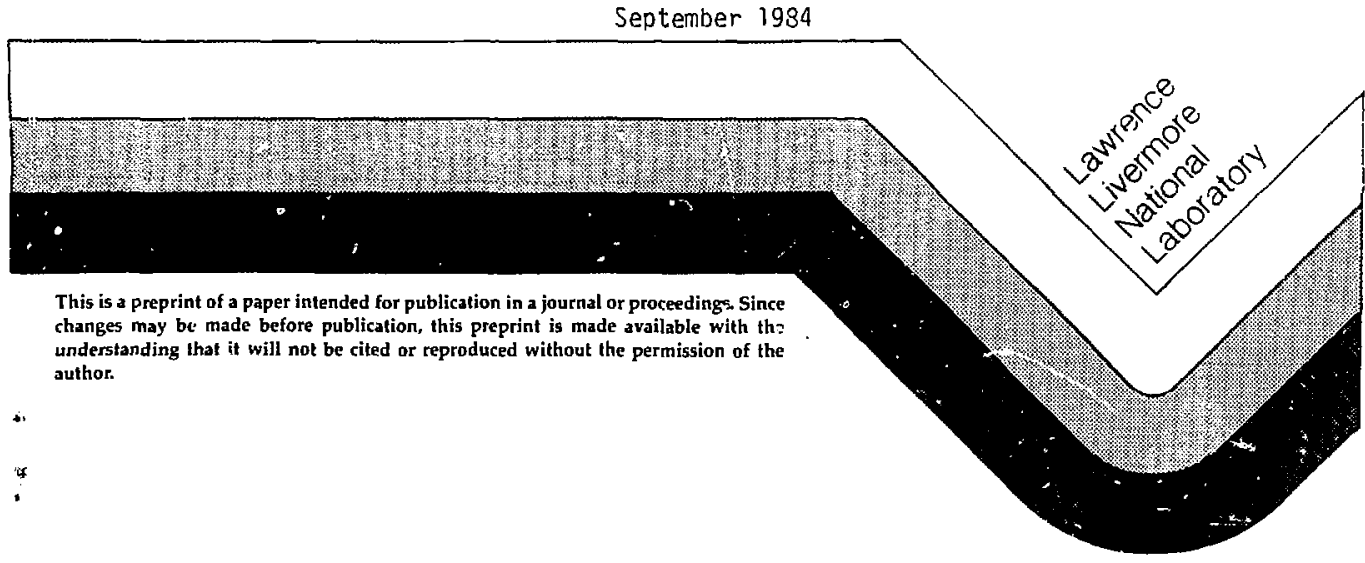


Low-Energy, High-Intensity Positron Beam Experiments with a Linac*

R. H. Howe11, M. J. Fluss, I. J. Rosenberg and P. Meyer

Lawrence Livermore National Laboratory, Livermore, Cal ifornia

UCFL -90935

DQS5 001048

Previous experiments with positrons from radionuclides have demonstrated that positron beams are a rich source of information about the surface condition of solids. We have now demonstrated the possibility of producing very intense beams at the Lawrence Livermore $100 \mathrm{MeV}$ electron 1 inac and installed an apparatus that produces a variable energy positron beam at energies between $500 \mathrm{eV}$ and $20 \mathrm{keV}$ with sufficient intensity to perform a variety of new positron experiments. The positron beam is pulsed with 10 ns to 3 microseconds duration at rates up to 1440 pulses per second, with as many as $10^{6}$ positrons available per pulse. Experiments that require either puised or steady currents are possible in an ultrahigh vacuum environment. For the first time two-dimensional angular correlation spectra of the surface positron and positronium annihilation at a single crystal sample have been obtained for copper.

\section{DISCLAIMER}

This report was prepared as an accoust of work sponsored by an agency of the United States Government. Neitl.w the United States Government nor any agency thereof, nor any of their employees, nakes any warranty, express or implied, or assumes any legal liability or respostsibility for the accuracy, completeness, or usefulness of any information, apparatus, product, or process disclosed, or represents that its use would not infringe privately owned rights. Reference herein to any specific commercial product, process, or service by trade name, trademark, manufacturer, or otherwise dow not necessarily constitute or imply its endorsement, recommendation, or favoring by the United States Government or any agency thereaf. The views and opinions of authors expressed herein do not necessarily state or reflect those of the United States Government or any agency thereof.

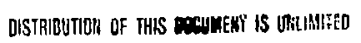




\section{Introduction}

The interaction of low energy positrons with surfaces can be used as a " surface probe. The surface-positron interaction is complex and several mechanisms for obtaining information about the surface are available by either following the positron history during the interaction or by measuring the characteristics of positrons annihilating with surface electrons. Because this is an emerging technology, the details of the physics of the positron-surface interaction are the subject of ongoing investigation, but several points are becoming clear. The most important physical effects are the ejection of bare positrons and positronium from surfaces due to their negative work function potentials and the trapping of positrons in their surface image potential (1).

Both the velocity of the ejected positronium and the angular correlation of the annihilation radiation from the positrons trapped at the surface can be related to the state of the participating electron. The interaction probability of the positron with a surface electron, along with conservation of energy and momentum, provides a direct relationship between the electron density of states and the energy distribution of ejected fositronium. There are several measurements of the energy distribution of positronium emitted from surfaces which have been determined by time of flight techniques (2). The overall characteristics of these measurements are in good agreement with the above description for the fast, high-energy component of the positronium distribution. Low energy positronium, however, is the result of a separate process whereby a position, or possibly positronium, trapped in the image potential at the surface, is thermally desarbed. 
The description of the correlation between the electronic states and the annihilation radiation of positrans in the surface trap is less ambiguous due to the recent availability of high resolution angular correlation data in two dimensions. The availability of similar data in bulk measurements has provided a clear description of features of the electron states such as the variation of the Fermi surface with crystallographic direction, or vacancy defect structure. Similar information about the electronic structure at the surface may be available from experimenis performed with a low energy positron bean.

\section{Positron Beam}

The investigation of surfaces with positrons depands on the availability of mono-energetic beams of positrons. The performance of several experiments of interest, including high resolution two dimensional angular correlation measurements, have been limited by the available intensity of positrons in such beams. The ejection of positrons by a negative work function is the mechanism used in all positron beam systems to obtain a beam with narrow energy width. The efficiency of positron ejection is dominated by the diffusion of implanted positrons to the surface and the size of the potential drop at the surface, while the eriergy spread of the positrons is mainly due to inelastic interactions near the surface. Increases in the intensity of beams were obtained by discovering better techniques for the preparation of negative work function moderator materials. However, further improvements must come from more intense sources of positrons. 
One intense source of energetic positrons is pair production from the electron beam of a linac. We have developed such a source at the $100 \mathrm{MeV}$ electron linac at Lawrence Livermore National Laboratory; first performing experiments that proved the principle and then defining a set of conditions that aliow the production of intense beams of positrons. The fiajor requirements found in intense beam production are the use of specially prepared tungsten foils as moderators in close contact with an electron radiator target of 2 to 3 radiation lengths thickness. Under these conditions $1 \times 10^{-6}$ positrons per electron are routinely available at $100 \mathrm{MeV}$ and factors of three greater can be achieved. The method of production of intense beams has been reported in detail in references $(3,4)$.

A facility has been installed at the Lawrence Livermore electron linac that provides positron teams over the full range of available electron beams. The apraratus consists of a positron production chamber located in a room that Lan receive the full linac beam and a transport system that magnetically guides the positrons into a UHV sample chamber. The sample chamber is located in a second room that is well shielded from the radiation produced at the positron production target. A schematic overview of this system, including the placement of position sensitive detectors (Anger cameras) for measuring the angular correlation of the annitilation gamma rays, is shown ill figure 1.

At the positron production chamber the energetic electron beam strikes a water cooled tungsten radiator $1 \mathrm{~cm}$ thick. To facilitace the removal of the radiator after activation by the electron beam, the radiator is contained in a small, separate vacuum container with $0.25 \mathrm{~mm}$ stainless steel windows for the 
incident electrons and emerging positrons. The moderator consists of an array of vacuum annealed tungsten foils mounted on an insulating standoff in the transport vacuum system with a $0.25 \mathrm{~mm}$ stainless steel window to allow entrance of the energetic pcsitrons. Positrons are accelerated from the potential of the moderator to grouno inrough one or more screens in order to minimize acceleration transverse to the magnetic guiding field. Positrons may be accelerated in this system from 0 to $20 \mathrm{keV}$. A schematic representation of the positron production apparatus is shown in figure 2 .

The magnetic transport system consists of a series of coils and solenoids arranged to provide guiding fieids up to 500 gauss. The constraints introduced by the shielding and room geometries result in a long positron flight path of $13 m$ that reidires a mixture of large radius coils at the positron production and sample chamber areas coupled to a small radius solenoid in between. In order to compensate for the earth's magnetic field and other local magnetic fields, there are dipole coils along all of the small diameter solenoids in both the horizontal and vertical planes. These coils are adjusted so that the positron beam is steered along the center of the transport system. Careful adjustment of each of the separate transport elements is necessary in order to achieve a positron beam that simultaneously has the properties of a small spot size, small transverse momentum, and high energy. Changing the beil: energy or position can result in large changes in the beam diameter, or unexpected shifts in the beam position if the cyclotron motion of the positrons in the guiding field is large. Under the best conditions, beam spots of $5 \mathrm{~mm}$ diameter can be obtained with shifts from the cyclotron motion contained within the spot diameter. 
The samples are contained in a UHV chamber having $2 \times 10^{-10}$ torr base pressure. Samples may be heated, Argon sputtered, and Auger electron analyzed. There is also a chevron micro-channel plate installed on ihe sample stage so the positron beam spot intensity and geometry can be observed during set up. Selected ports are covered by $0.25 \mathrm{~mm}$ stainless steel windows so that the annihilation gamma rays can escape the chamber with minimum attenuation for lifetime, time of $\mathrm{flight,}$ and angular correlation measurements.

The low energy positrons are produced directly from the electron beam at the linac so that the characteristics of the positron beam are related directly to the linac tuning modes. Sirice the electron current at a our linac can be several hundred microamps, we can generate moderated positron intensities that are greater than presently available from any other source. A table of linac tuning modes with the corresponding positron intensities is given below. The tabulated values were conservatively determined for a conversion efficiency of $1 \times 10^{-6}$; higher efficiencies have, however, been observed.

\section{Positron beam characteristics $\mathrm{e}^{+} / \mathrm{e}^{-}=1 \times 10^{-6}$}

$\begin{array}{lllll}\begin{array}{l}\text { pulse } \\ \text { rate } \\ \text { per second }\end{array} & \begin{array}{l}\text { pulse } \\ \text { length } \\ \text { microseconds }\end{array} & \begin{array}{l}\text { electron } \\ \text { current } \\ \text { microamps }\end{array} & \begin{array}{l}\text { positron } \\ \text { current } \\ \text { picoamps }\end{array} & \begin{array}{l}\text { positrons } \\ \text { per pulse }\end{array} \\ 300 & 3 & 400 & 200 \mathrm{a}) & 4 \times 10^{6} \\ 900 & 3 & 100 & 100 & 6 \times 10^{5} \\ 1440 & .020-.001 & 100 & 100 & 4 \times 10^{5}\end{array}$

Table 1. Electron and positron beam capabitities at the Livermore $100 \mathrm{MeV}$ electron linac. a) In this case the positron current is limited by the ability to dissipate heat in the electron radiator. 


\section{Experiments}

We have used this apparatus to perform measurements of the electron-positron momentum distribution for positrons trapped at the surface of a sample and of positronium decaying in flight. We have also performed measurements of the energy distribution of positronium ejected from the surface of clean copper samples by measuring the time-of-flight of positronium decaying in front of a well collimated detector and measured the amount of positronium produced from the time spectrum of all annihilation gamma rays.

The positron-electron pair-momentum was determined in a plane normal to the direction of the annihilation radiation by measuring the deviation from collinearity of the annihilatior: gamma-rays along both axes in the plane. Since the two photon annihilation gamma-rays are equal in energy and opposite in direction in the rest system of the annihilating pair, the deviations from collinearity result from the transformation of the center of mass of the noving pair into our laboratory frame. For a positron at rest the angular deviation is proportional to the momentum of the electron while for positronium the deviation is proportional to the positronium momentum.

Our angular correlation spectra were measured with a system similar in principle to that developed by West using position sensitive Anger camera gamma-ray detectors each consisting of a $13 \mathrm{~mm}$ by $400 \mathrm{~mm}$ inch NaI crystal connected to an array of 37 phototubes (3). The location of a gamm ray on the face of the crystal could be determined to within $8 \mathrm{~mm}$ so that with the detectors at $13.67 \mathrm{~m}$ we had an angular resolution of $0.9 \mathrm{mrad}$. Valid gamma-ray 
events were selected by requiring a coincidence between the two Anger camera detectors and the pulse of positrons from the linac. Positions and relative detection time for the gamma-rays in each detector for each event were stored on tape using a Nuclear Data $€ 660$ computer, while the angular correlation spectra, corrected for the geometric loss of detection efficiency for large angles, were formed on the Livermore multi-user CDC 7600 system.

The electron 1 inac was run with 3 microsecond wide pulses at 900 per second. The positron intensity was 1 imited by saturation in the detector system to about $5 \%$ of the maximum. Due to the high intensity of the positrons in the beam pulse both the accidental backgrounds and the loss of events due to pileut rejection are larger than for angular correlation systems using random positron sources. Pile-up events were rejected by taking the first event in the energy window of each detector for each beam pulse. Accidental coincidences were minimized by setting a narrow time window on the valid events. Events that satisfied all constraints but were outside the time window were also stored and processed into accidental angular correlation spectra that were then normalized and subtracted from the data as background.

- The sample in these measurements is a single crystal of copper oriented with the [121] axis along the beam direction and the [111] axis along the 7 ine joining the camera centers so that the data are resolved in the [111] plane. The sample surface was cleaned by Argon sputtering and annealing and analyzed by Auger electron spectroscopy. There was less than $10 \%$ surface contamination at the end of a $24 \mathrm{hr}$ run. 
Lontour plots of the data taken in this geometry with both positron energies of $18 \mathrm{keV}$ and $740 \mathrm{eV}$ are displayed in figure 3 . The top frame of the figure shows the data and a model of the distribution of positronium escaping the surface with a net momentum into the vacuum for the $18 \mathrm{keV}$ incident positron energy, while the bottom frame shows the data for the underlying distribution for both energies. The electrostatic potential of the samp?e was adjusted so positrons re-emitted from the sample were attracted back to the surface for the $740 \mathrm{eV}$ positron beam and not for the $18 \mathrm{keV}$ beam, to enhance the bulk nature of the $18 \mathrm{keV}$ beam data.

The data obtained with the $18 \mathrm{keV}$ positron beam are quantitatively similar to that taken with high energy positrons indicating that most of the positrons are annihilating in the bulk of the sample. Hcwever, the data obtained with $740 \mathrm{eV}$ positrons that result from the annihilation of positrons trapped in the ir image potential at the copper surface and positronium ejected from the surface annihilating in flight are very different. The momentum distribution of the positronium is determined by kinematics, the positron-electron interaction and the electron density of states. The model shown in comparison with th: data was calculated for a free election gas. The momentum distribution for the positrons trapped in the surface well shows a distinct asymmetry as the width parallel to the surface is narrower than that perpendicular to the surface. There are also relatively fewer annihilations with high momentum core electrons seen in the $740 \mathrm{eV}$ spectrum than in the high energy spectrum. These measurements have already answered questions regarding the positron-surface interaction and should develop into a prac ica? tool for the general study of surface phenomena (6). 
Another technique which makes use of the pulsed positron beam is to measur the energy distribution of the emitted positronium with a detector collimated so that only radiation from a small yolume in front of the sample is aciepted. We are making such measurements usirig a detector geometry similar to that reported by Mills (2). Figure 4 shows data obtained with the detector collimation set so that a volume $10 \mathrm{~cm}$ from the sample surface and $1 \mathrm{~cm}$ thick was sampled. The overall time resolution in the system, including the width of the positron beam, is $15 \mathrm{~ns}$ so that good energy resolution is obtained. High resolution energy measurements of the positronium energy can be directly related to the aistribution of the density of states for the electrons in the sample. The overall frartion of positronium produced also depends on the surface condition. He obtain a measurement of the positronium fraction by taking the ratio of long lived to promptly decaying positrons.

\section{Summary}

We have instalied an apparatus at the $100 \mathrm{MeV}$ electron 1 inac at Lawrence Livermore National Laboratory that routinely produces intense beams of low energy positrons for solid state and materials science experiments. At the linac we can transport the full range of moderated positron beams from a high radiation area to an area of reduced background. In the low background area there is an apparatus for sample preparation and measurement that includes an instrumented ultrahigh vacuum chamber suitable for surface analysis and on-1ine miasurement. Diagnostic apparatus for monitoring the positron-sample interaction and subsequent positron anniililation allows us to determine positronium formation strengths and energy spectra by time of flight. A two dimensiona: angular correlation system for high resolution measurements of the 
momentum of the annihilating electron-positron pair is in place for use with the bean. Preliminary data have been cbtained in these experiments promising new possibilities in the analysis of surface structure.

\section{Acknowledgments}

The help of M. Connor and L. Bernardez in collecting the data, A. Coombs in developing the instrumentation and $\mathrm{J}$. Kimbrough in data acquisition software and hardware development is gratefully acknowiedgea.

* This work was supported by the U.S. DOE, under contract number W-7405-ENG-48. 


\section{References}

1. A. P. Mills, Jr., Positron Solid-State Physics, W. Brandt and A. Dupasque eds. North-Holland New York p. 432, (1983).

2. A. P. Mills, Jr., L. Pfeiffer and P. M. Platzman Phys. Rev. Lett. 51, $1085(1983)$.

3. R. H. Howel1, 只 A. Alvärez and M. Stanek, App 1. Phys. Lett. 40, 751 (1982) and R. H. Howell, R. A. ATvarez, K. A. Woodie, S. Dhawan, P.

0. Egan, V. W. Hughes and M. W. Ritter, IEEe Trans, on Nuc. Sci. 30, $1438(1983)$.

4. G. Graff, R. Ley, A. Osipowicz, G. Werth and J. Ahrens, Appl. Phys, A 33, $59(1984)$.

5. R. N. West, J. Mayers and P. A. Walters, J. Phys. E 14, 478 (198.1).

6. R. H. Howell, P. Meyer, I. J. Rosenberg and M. L. Fluss, to be published. 


\section{Figure Captions}

Figure 1. Overview of the Livermore positron beam apparatus. The elertron linac beam enters from the right. The positrins are created, moderated and accelerated in the room at the lower right. The low energy positrons are then transported $13 \mathrm{~m}$ along a magnetic guiding field to the UHV sampla chamber. The Anger cameras used to measure the angular corre?ation in two dimensions are pictured at $12 \mathrm{~m}$ from the sample chamber.

Figure 2. Schenatic of the front end geometry in the Livermore positron beam system. Problems of shielding from residual radiation and cooling are simplified by keeping the radiator-converter assembly soparate from the vacuum. Both aluminum and stainless steel windows have bean used.

Figure 3. Contour plots of the positronium and surface bound positrons in the top and bottom frames respectively. The sample surface lies along $P_{y}$ and positive $p_{2}$ points into the sample. A model of the distribution of the free positronium obtained from a free electron gas provides a qualitative description of the positronium data. Positrons annihilating at the sample have very different spectra when at the surface, $740 \mathrm{eV}$, and in the bulk, $18 \mathrm{keV}$ indicating large differences in the momenta of the electrons participating in the annihilation. 
Figure 4. Positronium time-of-flight spectrum. Time runs from right to left and zero time is at the peak of the promp' annihilations. The flight path of the positronium was $9.5+8-0.5 \mathrm{~cm}$. The peak of the positronium oistribution is at $1.1+1-0.1$ el and the maximum positronium energy is at $2.6+1-0.3 \mathrm{eV}$ in good agreement with the negative positronium work function. 
LIVERMORE SYSTEM

$\stackrel{7}{\square}$

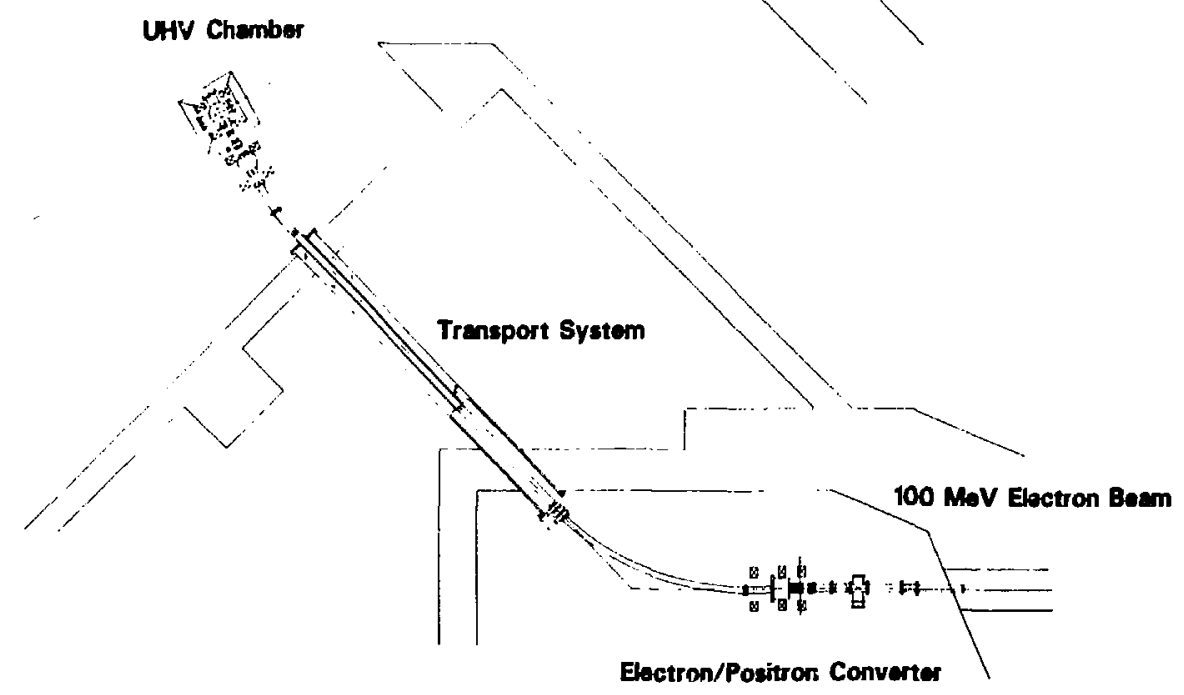




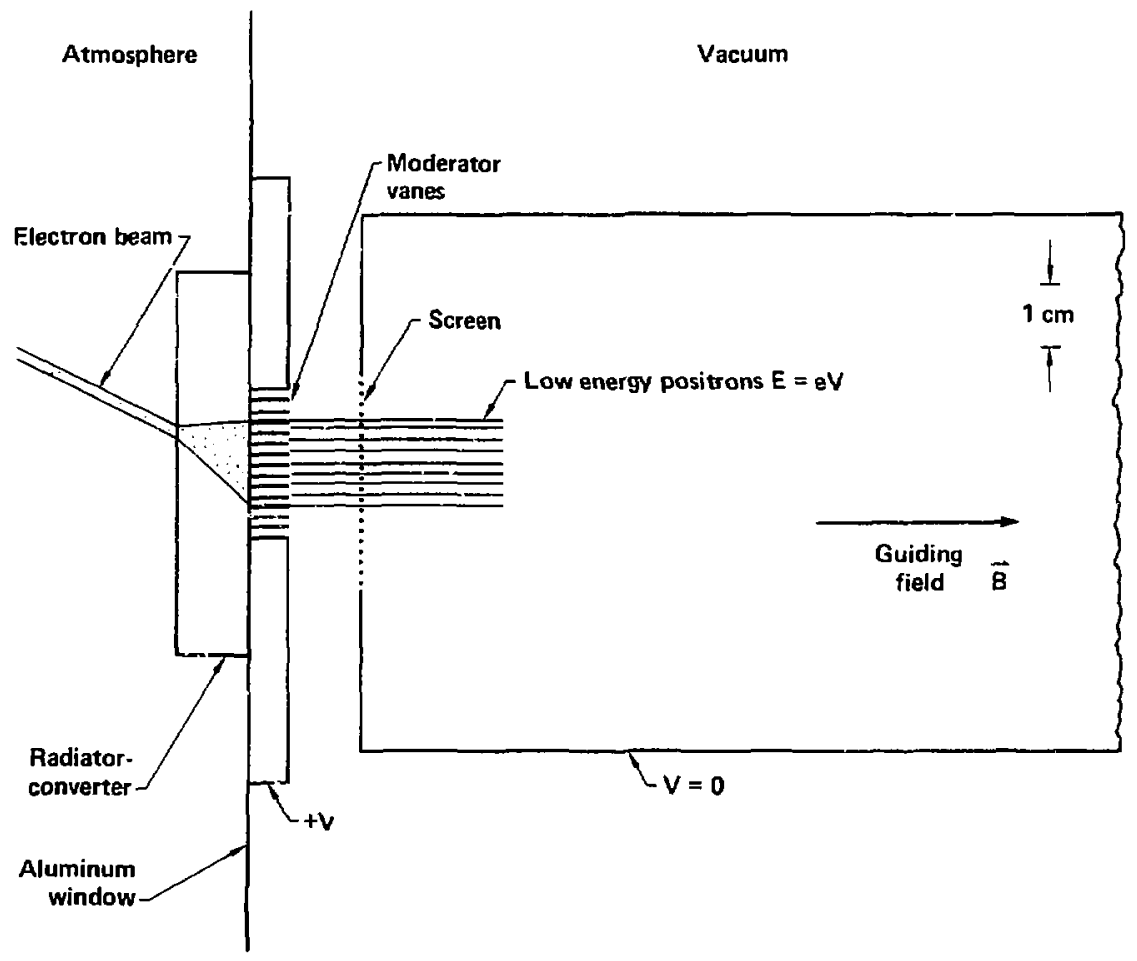

Fig. 2 

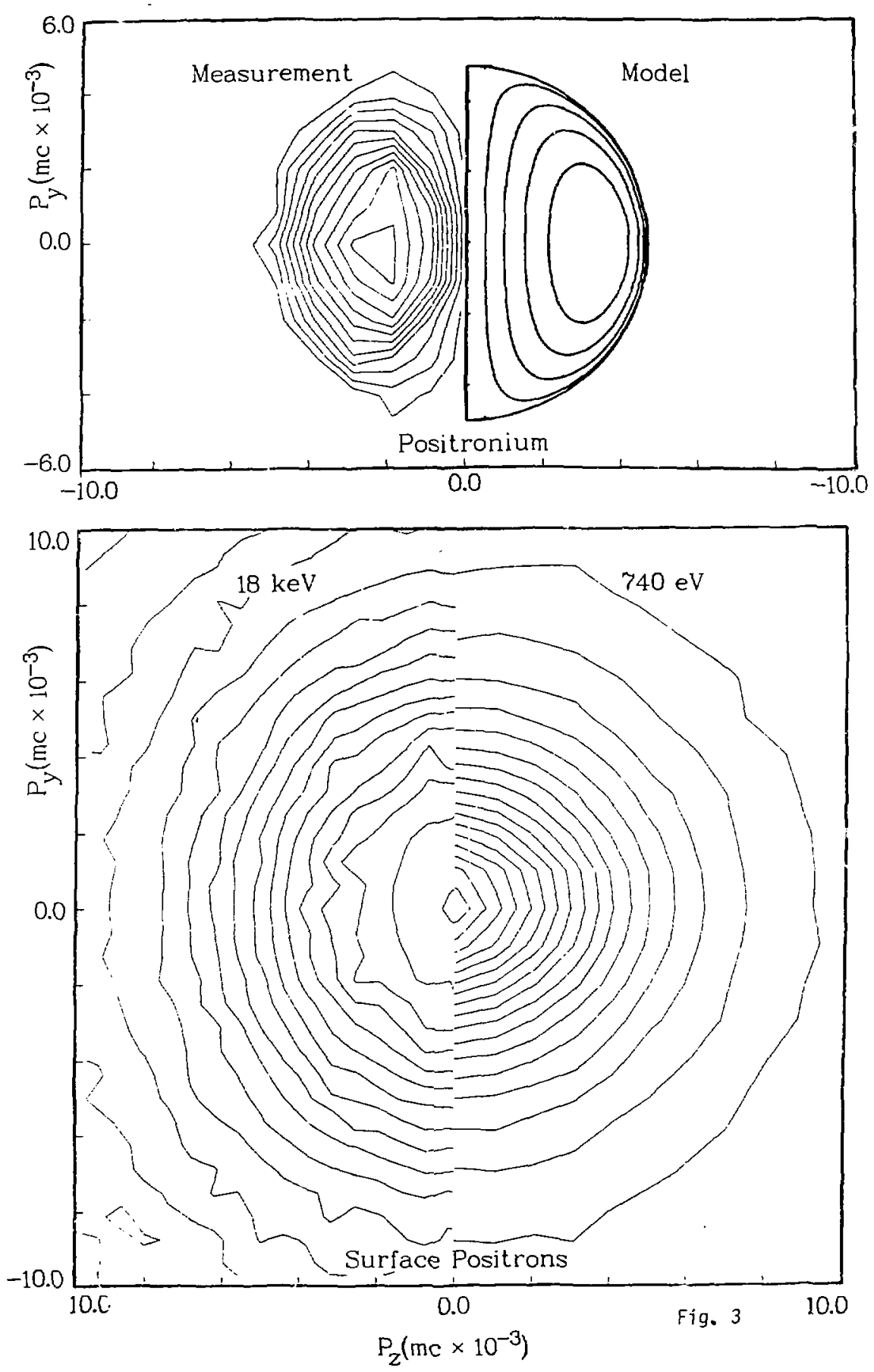